\title{
Time Series Analysis of COVID-19 Data to Study the Effect of Lockdown and Unlock in India
}

\section{Saswat Singh}

Jadavpur University

Chandreyee Chowdhury ( $\nabla$ chandreyee.chowdhury@gmail.com )

Jadavpur University

Ayan Kumar Panja ( $\square$ ayanpanja1992@gmail.com )

Institute of Engineering \& Management

\section{Sarmistha Neogy}

Jadavpur University

\section{Research Article}

Keywords: COVID-19, Coronavirus, Pandemic, Time-series, ARIMA, Trend, Forecast, India

Posted Date: September 29th, 2020

DOI: https://doi.org/10.21203/rs.3.rs-83179/v1

License: (9) This work is licensed under a Creative Commons Attribution 4.0 International License. Read Full License

Version of Record: A version of this preprint was published at Journal of The Institution of Engineers (India): Series B on April 8th, 2021. See the published version at https://doi.org/10.1007/s40031-02100585-7. 


\section{Time Series Analysis of COVID-19 Data to Study the Effect of Lockdown and Unlock in India}

Received: date / Accepted: date

\begin{abstract}
The ongoing COVID-19 pandemic has caused worldwide socioeconomic unrest, forcing governments to introduce extreme measures to reduce its spread. Being able to accurately forecast the effect of unlocking in India, would allow governments to alter their policies accordingly and plan ahead. The study investigated prediction forecasts using the ARIMA model on the COVID-19 data on the lockdown period and the unlock period. In this work, we have considered not only the no of positive COVID cases but also considered the number of tests carried out. The time-series data sample was collected till June 2020 and the prediction and analysis are done for August 2020. The model developed and the forecasted results align very closely with the actual no of cases and we have drawn some important inferences through experimentation.
\end{abstract}

Keywords : COVID-19 · Coronavirus · Pandemic . Time-series · ARIMA · Trend · Forecast · India

\section{Introduction}

COVID-19 is an infectious disease caused by the Coronavirus, biologically known as severe acute respiratory syndrome coronavirus 2 (SARS-CoV-2). The disease was first spotted in the capital of China's Hubei province, Wuhan district in December 2019 and has spread through out the world and became a global pandemic. The COVID19 cases are growing ever since it broke into India. Patients are suffering from respiratory failure from acute respiratory distress syndrome (ARDS) which is the major cause of death. The healthcare personals are one of the majorly affected individuals during this pandemic. A study of the characteristics of people affected in the

Address(es) of author(s) should be given healthcare domain was published by CDC covid19 response team in [5]. Various researches[12,11,6] are carried out to identify features of cases with novel Coronavirus disease(COVID 19). Significant progress has also been made in the field of vaccine development[10]. After the 74 days of lock down in India the government has issued Unlock 1 on June 8,2020 where various sectors of the businesses was allowed to run in a restricted manner. India is among a group of most risked countries where relaxing lock downs could lead to a spike in new infections. According to various analysis carried out it has been observed that one of the two cases can crop up due to the effects of unlocking. The first case can be if the number of cases declines and the growth curve for number of cases flattens a positive feedback loop will dive in. The second case can be that the reopening of the economy can accelerate the number of cases which in turn will cease mobility.

Analyzing the outcome of unlock condition should be the need of the hour research domain. In this work, we have explored various time series models for prediction of positive cases. Then, we employed Auto-Regressive Integrated Moving Average(ARIMA) model for analysing the unlocking effect and predicting the incidence of 2019nCov disease. The main objective of the paper is to identify the effect of unlocking in India by doing a comparative study on the forecasting using the unlock and the lock down COVID-19 data for both positive cases and the total no of tests conducted.

The paper is organized as follows. The related works are discussed in the next section. The proposed methodology is discussed in Section 3. Experimental results are reported in Section 4 while the subsequent section concludes. 


\section{Related Work}

Few recent papers could be found on the analysis of infection spreading pattern in different countries [13]. There have been a lot of previous study that employed machine learning and statistical approaches to capture the patterns and trends of a number of varying events related to infectious diseases [16]. Works can also be found on application of SIR models [1] for contact tracing and prediction of positive no of cases. However, a common assumption of such works is to treat the density of the Indian population to be homogeneous and not considering the no of tests conducted.

Time series analysis is popularly used to forecast different diseases such as SARS, Ebola, pandemic influenza and dengue. Tandon et al. in [17] has performed a study by using $\operatorname{ARIMA}(2,2,2)$ model to predict the future number of COVID-19 cases in India and have drawn conclusion that cases in India will increase exponentially and predicted that the cases to decline from their anticipated forecast. They had reflected the importance of social distancing and sanitization in effort to decrease human exposure to the Virus. The motive of their study was to help the government and medical workforce to be prepared for the situation. The drawback of their study was that they had not taken into consideration the effect of number of COVID-19 test that resulted in positive cases and were unable to reflect to the point of asymptomatic cases and its effect on the spread of the virus.

Another similar study was done by Tyagi et al. in [18] where they concluded to have predicted the number of COVID-19 cases till end of month June and predicted the requirement of ICU beds, ventilators and isolation beds in India using ARIMA(1,3,1) model. The main objective of their work was to predict the medical requirement (ICU $10 \%$ of the Active cases, BEDS, Ventilators for $5 \%$ of the active cases and isolation bed). So, in this paper we aim at studying the effect of lockdown and unlock data using time-series prediction models considering the no of tests conducted corresponding to the no of positive cases. This is detailed in the next section.

\section{Methodology Description}

\subsection{Dataset Details}

The no of Confirmed cases, recovered cases and death cases of COVID-19 infection are collected for India from the official website of pre legislative research from 12 th march 2020 to 2nd July 2020 (https://prsindia.org/COVID19 /cases? search-box $=700023$ ). This data is used to build predictive model for analysis.

\subsection{Description of the Time Series Models under} Consideration

Time series is a series of data points indexed (or listed or graphed) in time order. Therefore, the data is organized by relatively deterministic timestamps and may be compared to random sample data containing additional information that we can extract. Over the past several decades, a lot of effort and research output has been produced towards the development and improvement of time series forecasting models. In this work we have tested seven different forecasting methods which are presented and analysed on the accuracy of the prediction for accurate selection of model.

\subsubsection{TBAT}

TBATS [3] is an acronym for key features of the model:

- T: Trigonometric seasonality

- B: Box-Cox transformation

- A: ARIMA errors

- T: Trend

- S: Seasonal components

TBATS model has the capability to deal with complex seasonality (e.g., non-integer seasonality, non-nested seasonality and large-period seasonality) with no seasonality constraints, making it possible to create detailed long-term forecasts. TBATS makes it easy for users to handle data with multiple seasonal patterns. This model is preferable when the seasonality changes over time.

In case of COVID-19 data, we had to choose from various models such as box-cox transformation or with or without trend or non-seasonal model and various amounts of harmonics used to model seasonal effects or with and without $\operatorname{ARMA}(\mathrm{P}, \mathrm{Q})$ process used to model residuals. Firstly, the data plots are looked into for any seasonal pattern, but the data did not show any seasonal pattern. To further investigate for any traces of seasonality in the COVID-19 data, we redefined the data and fit it into TBATS model taking two length of seasonality period, one of a fortnight and one over a month. Based on our findings, the trend has been modelled with $[0,0]$ seasonal harmonics. TBATS method is very generic. Under the hood, it builds and evaluates many model candidates. This results in making the processing slower. Although TBAT is usually more useful 
when one needs to train models for numerous parallel time series.

\subsubsection{Prophet}

Prophet[19] is a procedure for forecasting time series data based on an additive model where non-linear trends are fit with yearly, weekly, and daily seasonality, plus holiday effects. Prophet is robust to missing data and shifts in the trend, and typically handles outliers well. Prophet requires time series data to have a minimum of two columns- the time stamp and the values. With just a few lines, Prophet can make a forecast model every bit as sophisticated as the ARIMA model. We had forecasted till the month of August(frequency considered weekly) by using Prophet. The method uses an easily decomposable time-series model consisting of three main components: trend, seasonality and holidays. To forecast trend, a piece-wise linear model is used because reaching a saturation point in the near future is not feasible until a vaccine is developed. Since the growth rate in India has been varying over 2-3 percent over the period of lockdown, so it is taken to be constant for that period. Seasonality is taken as false. Holidays, as implemented in Prophet, can be thought of as unnatural events when the trend will deviate from the baseline but return once the event is over as in the case of different phases of lockdown and unlock in India.

\subsubsection{Auto-regressive Integrated Moving Average (ARIMA)}

In every time series analysis the forecast is solely based on the past values of the series called lags. A simple overview of a model that depends on one lag or one variable in the series is depicted in equation 1.

$Y_{t}=\omega+\phi \times Y_{t-1}+e_{t}$

Here, the predicted value, $Y_{t}$ depends on the previous prediction, $Y_{t-1}$ and the error $e_{t}$ calculated as the difference between the predicted and actual outcome. $\phi$ is the slope coefficient and $\omega$ is the non zero mean. The ARIMA model [7] is one of the models which directly doesn't depends on the lags or variables of the times series but depends on the error lags which is estimated by subtracting the actual outcome from the forecasted outcome. ARIMA models assume a linear correlation between the time-series values and attempt to exploit these linear dependencies in observations, in order to extract local patterns, while removing high-frequency noise from the data.

\subsubsection{Moving Average}

Moving averages[2] are a simple and common type of smoothing used in time series analysis and time series forecasting. Calculating a moving average involves creating a new series where the values are comprised of the average of raw observations in the original time series moving average of order $m$; where $m=2 k+1$. The estimate of the trend-cycle at time $t$ is obtained by averaging values of the time series within $k$ periods of $t$. The average eliminates some of the randomness in the data, leaving a smooth trend-cycle component. With respect to the COVID-19 data, it helps to figure out the trend in the data. We take the period of 5-7 days as the number of tests keeps increasing and trend keep changing with respect to time.

\subsubsection{Neural Basis Expansion Analysis (N-BEATS)}

N-Beats [14] is a times series forecasting model that employs a deep neural architecture consisting of forward and backward residual links along with a deep stack of fully-connected layers. Generally, the model operates in the same way as traditional decomposition techniques, such as, the seasonality-trend-level approach. More specifically, the architecture is comprised of two stacks: the trend stack, followed by the seasonality stack, each consisting of several blocks connected using residual connections. From the lookback period, the model learns the behaviour and predicts future points in the forecast Period. This input is passed through layers of stacks. We set the forecast period to 34 data points and lookback period of 88 data points.

\subsubsection{Single exponential method}

Simple Exponential Smoothing is a time series forecasting method for uni-variate data without a trend or seasonality. It requires a single parameter, called alpha $(\alpha)$, also called the smoothing factor. This parameter controls the rate at which the influence of the observations at prior time steps decay exponentially. $\alpha$ is often set to a value between 0 and 1 . This method was used for the analysis as the raw data itself does not show any clear trend or seasonal pattern.

\subsubsection{Double exponential method}

Double Exponential Smoothing [9] is an extension to Exponential Smoothing that explicitly adds support for trends in the univariate time series. Apart from $\alpha$, another factor, $\beta$ is added to control the decay of the influence of the change in trend. The method supports 
trends that change in both additive (linear trend) and multiplicative (exponential trend) way.

Double exponential method had an upper edge compared to single exponential method because a change in trend is observed in the COVID-19 data. A change from linear trend to exponential trend to linear trend was observed.

\subsection{Model Selection}

All the models are executed on the Covid-19 dataset and the errors are listed in Table 1. It has been observed from the Root Mean Square error (RMSE) measures that the ARIMA model $[15,8]$ has the best forecasting accuracy. So, we have used ARIMA based model to develop relationship between the lockdown and the unlock data and perform forecasting on the time series data.

Table 1 RMSE values corresponding to the performance of different models on the Covid-19 data

\begin{tabular}{|c|c|}
\hline Model Name & RMSE \\
\hline TBAT & 0.005642 \\
\hline Prophet & 0.098641 \\
\hline ARIMA & 0.004897 \\
\hline Moving Average & 0.018425 \\
\hline -BEATS & 0.251691 \\
\hline Single Exponential Method & 0.022187 \\
\hline Double Exponential Method & 0.020894 \\
\hline
\end{tabular}

\subsection{Model development}

ARIMA model[4] is represented in the form of $P, D, Q$. Here, $P$ stands for the order of auto-regression, $D$ signifies the degree of trend difference and $Q$ is the order of moving average. Here, ARIMA model is applied to the time series data of confirmed COVID-19 cases in India. Auto correlation function (ACF) graph and Partial Auto correlation (PACF) graph are used to find the initial number of ARIMA models. These ARIMA models are then tested for variance in normality and stationarity.

Two tests are conducted to check for the stationarity of the series, Dickey-fuller test and rolling statistics. In order to get a stationary series, a mathematical transformation in the series is applied as follows.

$Y_{t}=\log (X) ; \quad Y_{t}=Y_{t}-Y_{t-m}$

Here, $\mathrm{X}$ is the representation of the data, $\mathrm{m}$ is Lag differences. Dickey-fuller and rolling statistics test gave the result that showed that the transformed data is stationary. The next step is to check for the ACF and PACF plots to get the value of $P$ and $Q$.

1. $P$ : The lag value where the PACF chart crosses the upper confidence interval for the first time $(P=2$ for lockdown and $P=1$ for unlock)

2. $Q$ : The lag value where the ACF chart crosses the upper confidence interval for the first time $(Q=2$ for lockdown and $Q=1$ for unlock)

It has been observed that forecasting of positive cases considering only number of positive cases increased the error deviation. Hence, both the number of positive cases and the number of tests conducted are considered for future prediction and analysis. With these two considerable lags, our prediction model is developed. The number of forecasted test data for a single day was used to develop a chart for the number of positive COVID19 cases with respect to the number of tests conducted. An average of the per day positivity rate (percentage of positive cases w.r.t the total tests conducted) was considered. We mapped the predicted value of positive cases by the ARIMA model based on the positive cases already recorded and the predicted chart by the ARIMA model based on the reported number of tests carried out per day and the average positivity rate. In order to get the total number of cases for the very day $d$, we considered an average of the predicted chart and the estimated positive cases which was added with the number of cases recorded till $(d-1)$ th day.

\section{Results and Observation}

A detailed overview of the ARIMA model used along with the parameters for lockdown and unlock is listed in Table 2 and 3 respectively.

Table 2 ARIMA MODEL RESULT ON LOCKDOWN DATA

\begin{tabular}{c|c|c|c} 
Dep. Variable: & D.Confirmed Cases & No. Observations: & 76 \\
Model: & ARIMA (5, 1, 4) & Log Likelihood: & 147.201 \\
Method: & css-mle & S.D. of innovations: & 0.030 \\
Date: & Wed, 15 Jul 2020 & AIC: & -272.402 \\
Time: & $13: 03: 25$ & BIC: & -246.764 \\
Sample period: & $24-3-2020$ & HQIC: & -262.156 \\
& to 07-06-2020 & &
\end{tabular}

In Fig 1, a line plot of the residual errors is presented. It suggests that there may still be some trend information not captured by the model.

In Fig 2, we have plotted a density plot of the residual error values. It can be observed from the plot that the residual error is distributed in a Gaussian manner which is centered around 0 . This indicates that the 
Table 3 ARIMA MODEL RESULT ON UNLOCK DATA

\begin{tabular}{c|c|} 
Dep. Variable: & D. Confirmed Cases \\
Model: & ARIMA $(1,1,1)$ \\
Method: & css-mle \\
Date: & Wed, 15 Jul 2020 \\
Time: & $12: 51: 35$ \\
Sample Period: & 06-10-2020 \\
to 07-15-2020
\end{tabular}

\begin{tabular}{c|c} 
No. Observations: & 36 \\
Log Likelihood: & 185.340 \\
S.D. of innovations: & 0.001 \\
AIC: & -362.679 \\
BIC: & -356.345 \\
HQIC: & -360.468
\end{tabular}
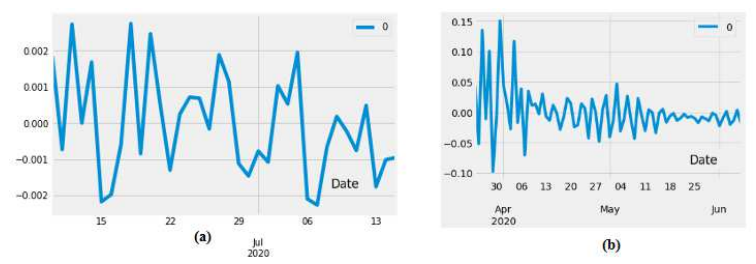

Fig. 1 (a)Error residual graph for unlock (b)Error residual graph for lockdown

model can be used for analysis of the COVID-19 dataset under consideration. The forecast results are presented in Fig. 3 along with the original plot to compare the two.
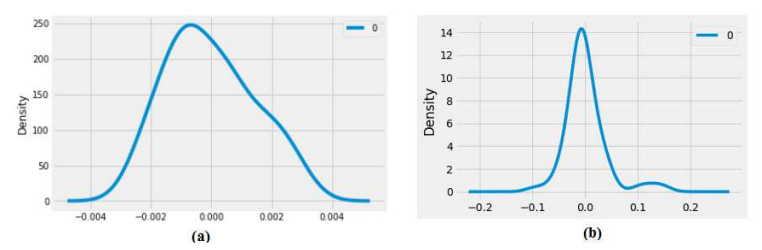

Fig. 2 (a)Density Plot error for unlock (b)Density Plot error for lockdown
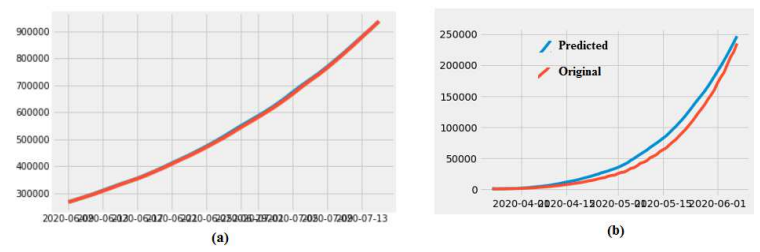

Fig. 3 (a)Prediction $\mathrm{v} / \mathrm{s}$ original positive cases for unlock period (b)Prediction $\mathrm{v} / \mathrm{s}$ original positive cases during lockdown in India

In Fig 3, it can be observed that the predicted data plot for both the unlock and the lockdown data align with the actual result. The two ARIMA models are also used for forecasts as shown in Fig. 4. For the lockdown data, we see a graph with $95 \%$ confidence interval that signifies a sharp increase in COVID-19 positive cases. The slope of the graph signifies the magnitude of the increase in cases per day. If the predicted graph is compared with the plotted graph of the number of COVID-19 cases post-lockdown, the same linear plot as the $\operatorname{ARIMA}(5,1,4)$ model is predicted.
From the above plots the following observations could be made.

1. The Unlock period is not the only factor for the increase in COVID-19 cases. There are numerous other lags in the system. The analysis on the lockdown model predicted the same trend in positive COVID-19 cases. This brings up some important questions: (a)Did the spread already occur in the community during lockdown? Was the lockdown effectively carried out to stop the virus spread? This question arises as some of the study suggests that among the total Coronavirus tests conducted so far, $69 \%$ were asymptomatic cases and $31 \%$ were symptomatic cases. So, another question also arises on the fact that the first cases detected in India was on 30th January, hence, could it be possible that the virus had spread before the very date?

2. If unlock period were the prime factor in increase of COVID-19 cases, then we would have witnessed noises and spikes in the unlock graph on the number of cases. The only difference that we came across is that the slope of Fig 4 and Fig 5 is that the slope of graph in Fig 5 is more steeper than that of Fig 4(a). So, the steeper slopes states that the spread of COVID-19 is fast and the cases will not slow over time as per the forecasted result and the change in slope is the result of increase in number of COVID19 tests which contribute in more cases detected per day .

With the number of tests increasing per-day, an increase in the number of cases could be observed. According to the data, around $10-12 \%$ of total tests were positive COVID-19 cases (Fig6). So, in the proposed model, both predictions based on no. of positive cases and no of tests conducted are merged to reduce the errors.

According to the proposed model, 518082 total tests and 50,234 positive cases were predicted on 31st July 2020. The very model has been extended to predict till August 14th as shown in Fig 7. It can be observed that the predicted and the actual values align and the average positivity rate stays within $7-11 \%$ both during the lockdown and the unlock.

The analysis made on both the lockdown data and the unlock data does not guarantee community spread in India. However, region wise community spread can be a possibility. So, the government must organize mass testing in regions where many COVID-19 patients are found. 


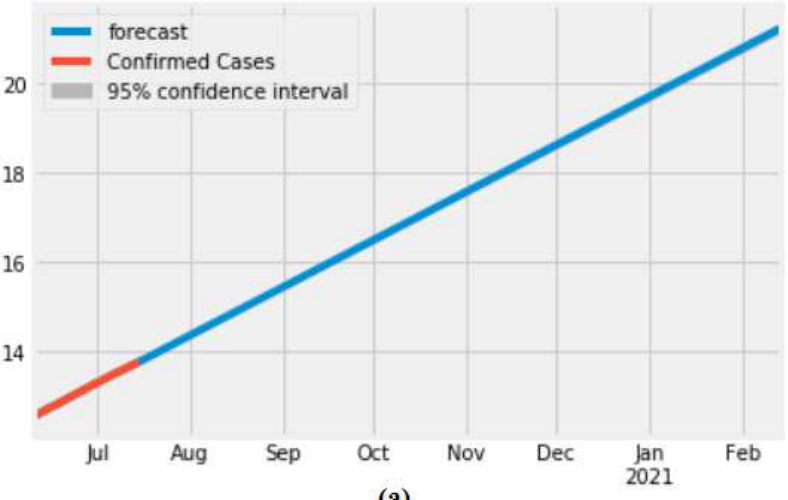

(a)

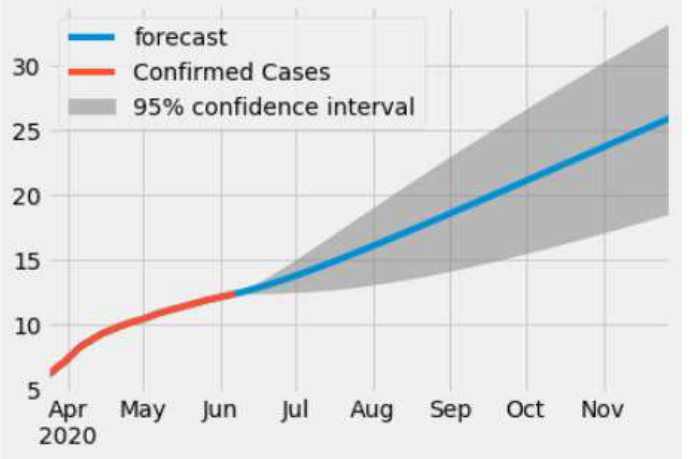

(b)

Fig. 4 (a)Forecasted result for unlock (b)Forecasted result for lockdown

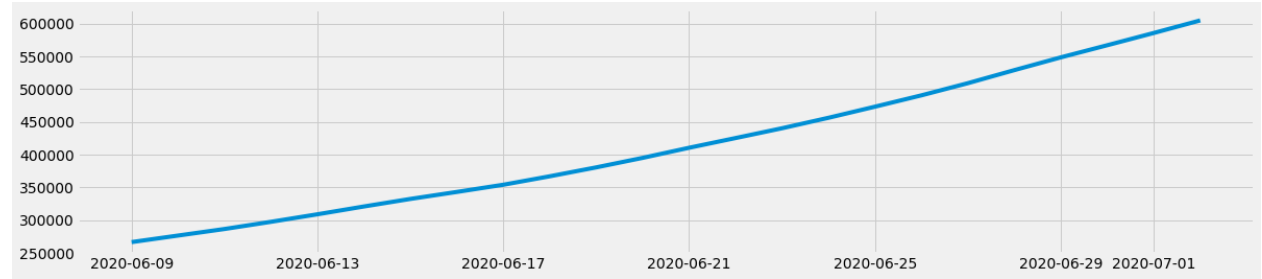

Fig. 5 No. of COVID-19 positive cases predicted for the unlock period

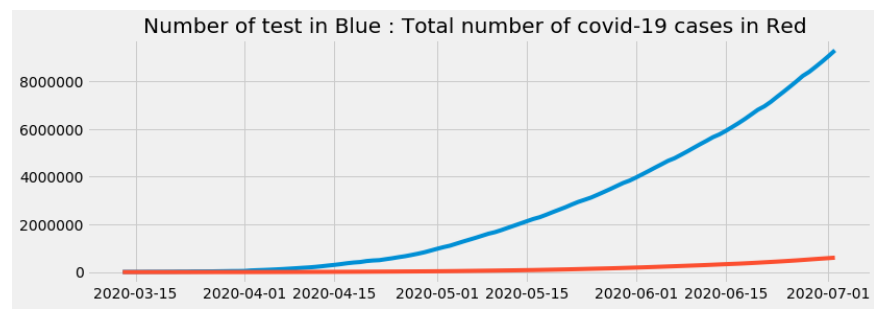

Fig. 6 Number of tests conducted VS Number of positive COVID-19 Cases

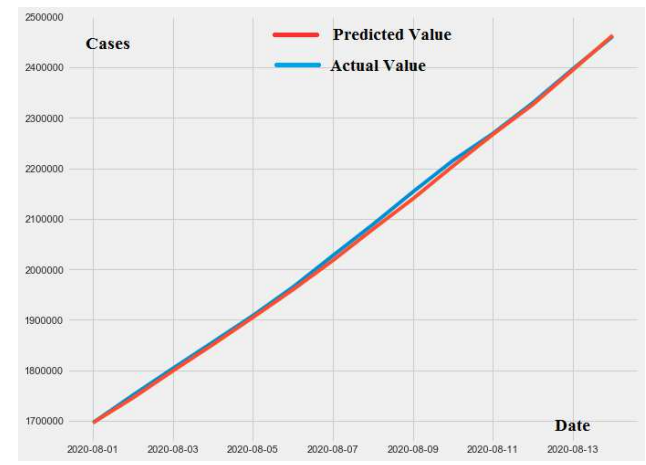

Fig. 7 Number of Positive cases predicted by the proposed model vs Actual cases, from 1st Aug to 14th Aug,2020

\section{Conclusion}

This paper investigates the applicability of different time series models to analyze COVID-19 data for India during lockdown and unlock. An ARIMA based predic- tion model is developed that makes prediction taking into account the no of positive cases, no of tests conducted and the average positivity rate. In the present circumstances, the proposed model could be valuable in anticipating future cases of infection if the pattern of virus spread did not change abnormally. The analysis showed that the increase in number of cases per day that shoot up after lockdown was not an abnormal trend. The predicted graph based on the lockdown data had showed a significant increase in number of COVID-cases. With the ARIMA model, the forecasts are produced on the prior values of the time series and the error lags which actually helps the model to adjust its prediction values from sudden change in trends. As we are interested in making short term forecasting in the time series and the outcome in the very near months, the ARIMA proved to be the most suitable model unlike N-Beats which requires a large amount of previously estimated timestamp samples.

The future modifications to further improve the predictive accuracy of the models will include the creation of ensembles of the presented models that would combine the best of many worlds in order to reduce the overall error as well as the adoption of multivariate time series modeling that take into account other factors that are either directly or indirectly related to the spread of the pandemic. Another future ambition would be to use some form of transfer learning in order to bring learn- 
ing's from one country to another in order to know the majority parameters for the actual cause of the spread.

\section{References}

1. Acemoglu, D., Chernozhukov, V., Werning, I., Whinston, M.D.: A multi-risk sir model with optimally targeted lockdown. Tech. rep., National Bureau of Economic Research (2020)

2. Box, G.E., Pierce, D.A.: Distribution of residual autocorrelations in autoregressive-integrated moving average time series models. Journal of the American statistical Association 65(332), 1509-1526 (1970)

3. BROŻYNA, J., Mentel, G., Szetela, B., Strielkowski, W.: Multi-seasonality in the tbats model using demand for electric energy as a case study. Economic Computation \& Economic Cybernetics Studies \& Research 52(1), 229246 (2018)

4. Contreras, J., Espinola, R., Nogales, F.J., Conejo, A.J.: Arima models to predict next-day electricity prices. IEEE transactions on power systems 18(3), 1014-1020 (2003)

5. COVID, T.C., et al.: Characteristics of health care personnel with covid-19-united states, february 12-april 9, 2020. MMWR Morb Mortal Wkly Rep. 2020 69(15), $477-481(2020)$

6. Fauci, A.S., Lane, H.C., Redfield, R.R.: Covid19-navigating the uncharted. New England Journal of Medicine 382(13), 1268-1269 (2020). DOI 10.1056/NEJMe2002387

7. Hillmer, S.C., Tiao, G.C.: An arima-model-based approach to seasonal adjustment. Journal of the American Statistical Association 77(377), 63-70 (1982)

8. Kalpakis, K., Gada, D., Puttagunta, V.: Distance measures for effective clustering of arima time-series. In: Proceedings 2001 IEEE international conference on data mining, pp. 273-280. IEEE (2001)

9. LaViola, J.J.: Double exponential smoothing: an alternative to kalman filter-based predictive tracking. In: Proceedings of the workshop on Virtual environments 2003, pp. 199-206 (2003)

10. Le, T.T., Andreadakis, Z., Kumar, A., Roman, R.G., Tollefsen, S., Saville, M., Mayhew, S.: The covid-19 vaccine development landscape. Nat Rev Drug Discov 19(5), 305-306 (2020)

11. Mehta, P., McAuley, D.F., Brown, M., Sanchez, E., Tattersall, R.S., Manson, J.J., Collaboration, H.A.S., et al.: Covid-19: consider cytokine storm syndromes and immunosuppression. Lancet (London, England) 395(10229), 1033 (2020)

12. Nishiura, H., Oshitani, H., Kobayashi, T., Saito, T., Sunagawa, T., Matsui, T., Wakita, T., COVID, M., Suzuki, M.: Closed environments facilitate secondary transmission of coronavirus disease 2019 (covid-19) (2020). DOI https://doi.org/10.1101/2020.02.28.20029272

13. Novel, C.P.E.R.E., et al.: The epidemiological characteristics of an outbreak of 2019 novel coronavirus diseases (covid-19) in china. Zhonghua liu xing bing xue za zhi= Zhonghua liuxingbingxue zazhi 41(2), 145 (2020)

14. Oreshkin, B.N., Carpov, D., Chapados, N., Bengio, Y.: N-beats: Neural basis expansion analysis for interpretable time series forecasting (2019)

15. Sato, R.C.: Disease management with arima model in time series. Einstein (Sao Paulo) 11(1), 128-131 (2013)
16. Shinde, G.R., Kalamkar, A.B., Mahalle, P.N., Dey, N., Chaki, J., Hassanien, A.E.: Forecasting models for coronavirus disease (covid-19): A survey of the state-of-the-art. SN Computer Science 1(4), 1-15 (2020)

17. Tandon, H., Ranjan, P., Chakraborty, T., Suhag, V.: Coronavirus (covid-19): Arima based time-series analysis to forecast near future. arXiv preprint arXiv:2004.07859 (2020)

18. Tyagi, R., Dwivedi, L.K., Sanzgiri, A.: Estimation of effective reproduction numbers for covid-19 using real-time bayesian method for india and its states (2020). DOI 10.21203/rs.3.rs-45937/v1

19. Zhao, N., Liu, Y., Vanos, J.K., Cao, G.: Day-of-week and seasonal patterns of pm2. 5 concentrations over the united states: Time-series analyses using the prophet procedure. Atmospheric Environment 192, 116-127 (2018) 
Figures

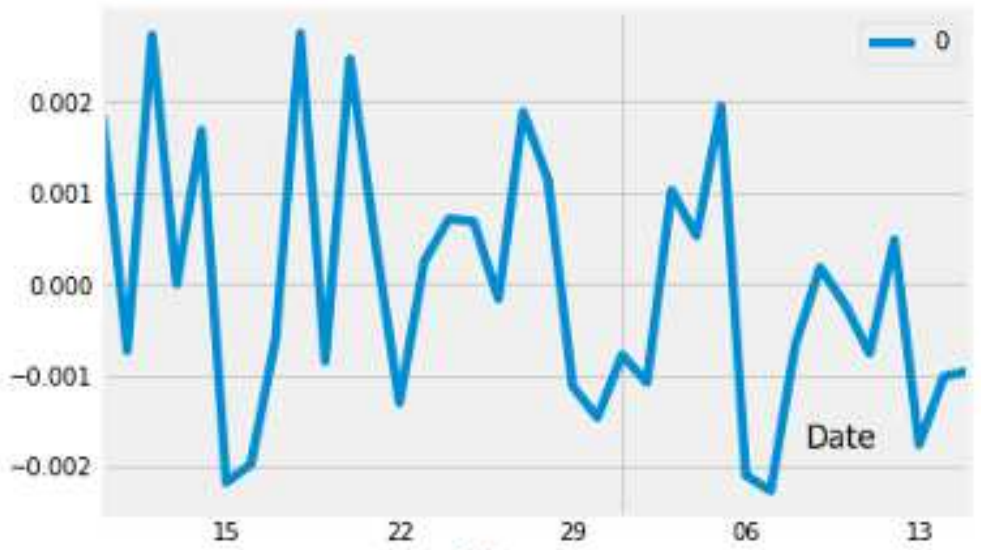

(a)

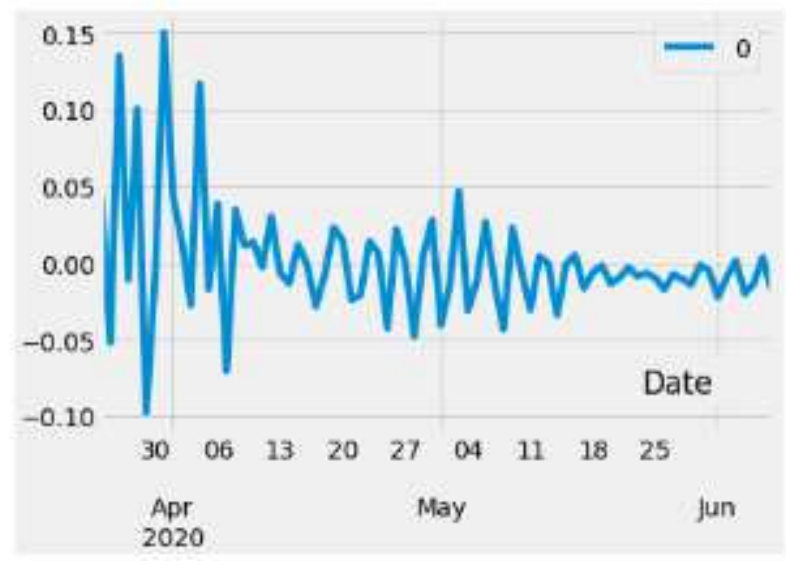

(b)

Figure 1

(a)Error residual graph for unlock (b)Error residual graph for lockdown

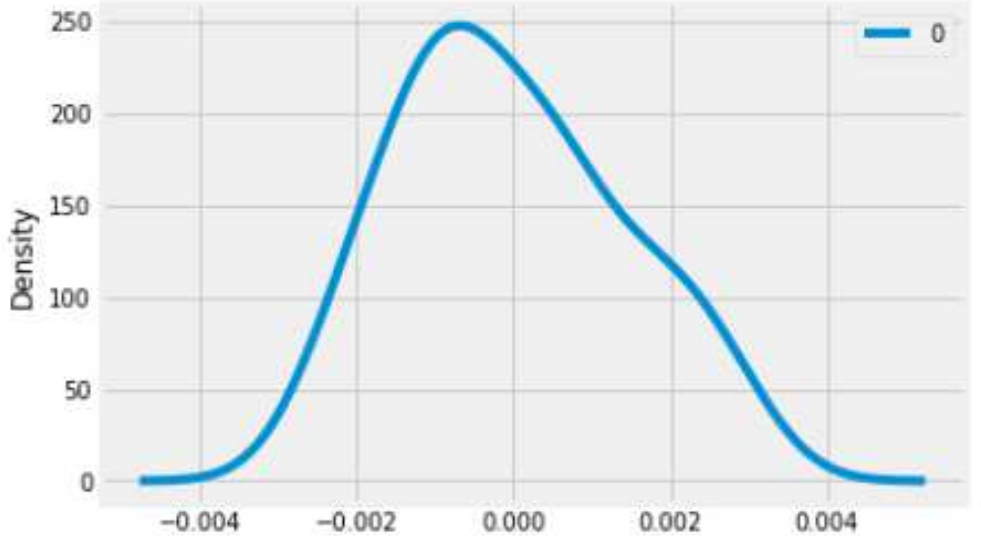

(a)

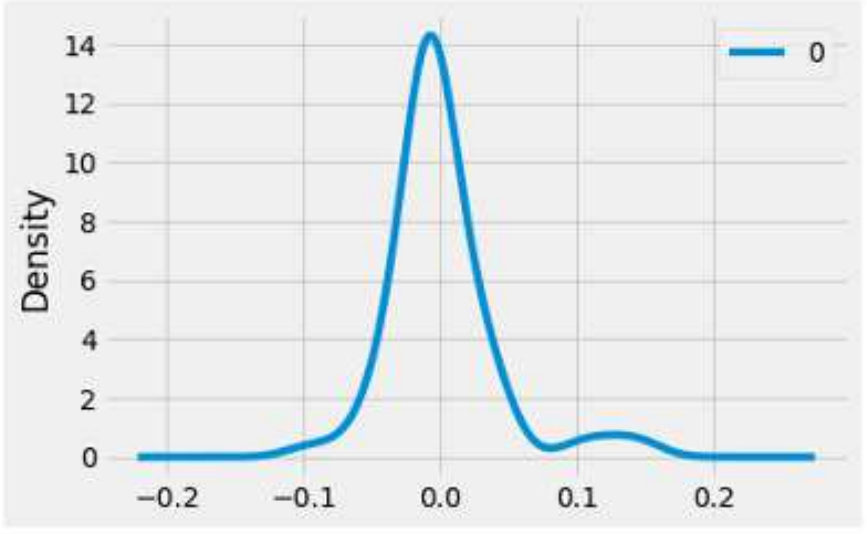

(b)

Figure 2

(a)Density Plot error for unlock (b)Density Plot error for lockdown 


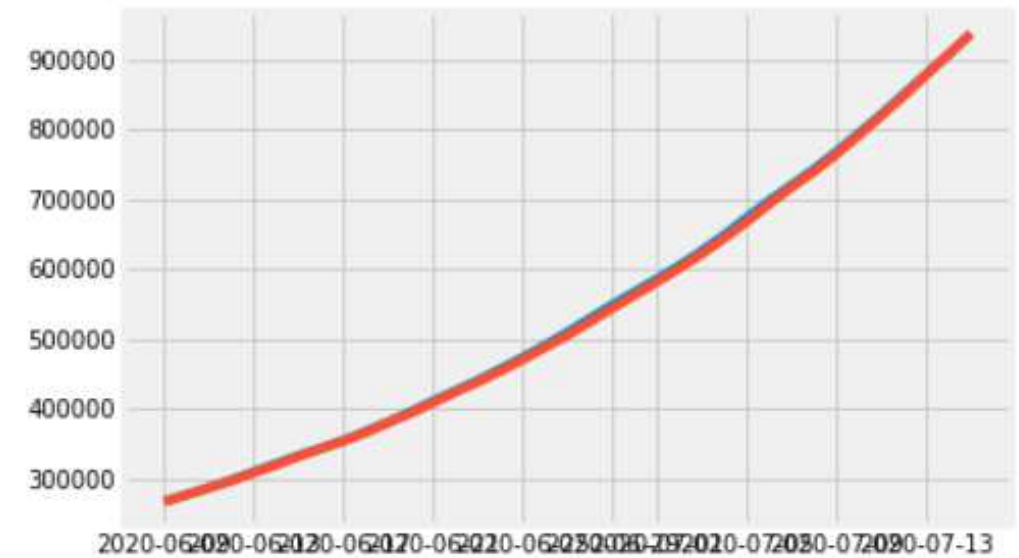

(a)

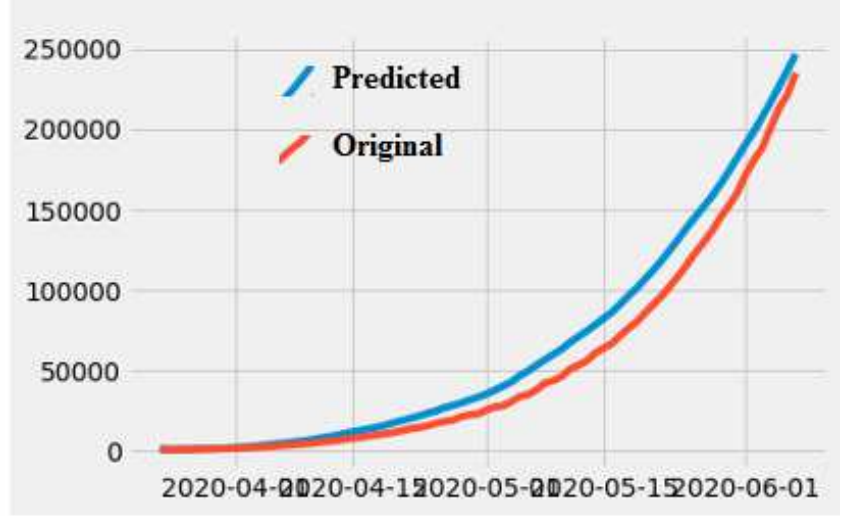

(b)

\section{Figure 3}

(a)Prediction v/s original positive cases for unlockperiod (b)Prediction v/s original positive cases during lock-down in India

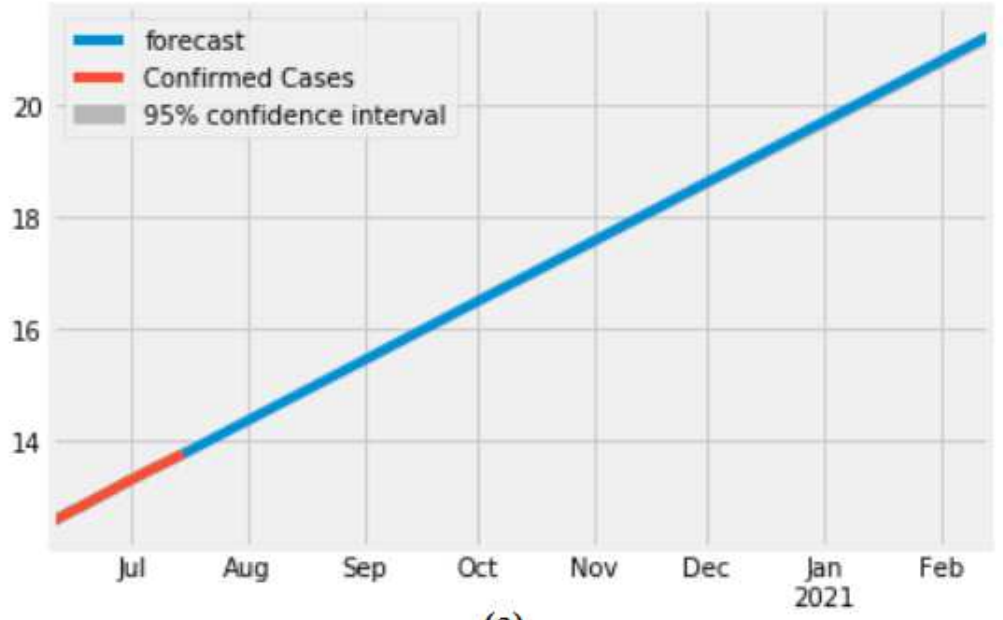

(a)

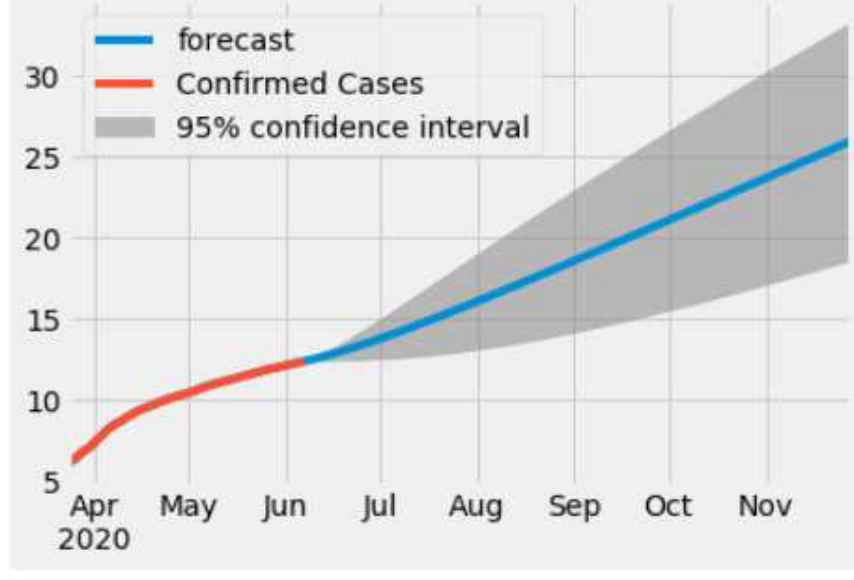

(b)

\section{Figure 4}

(a)Forecasted result for unlock (b)Forecasted result for lockdown

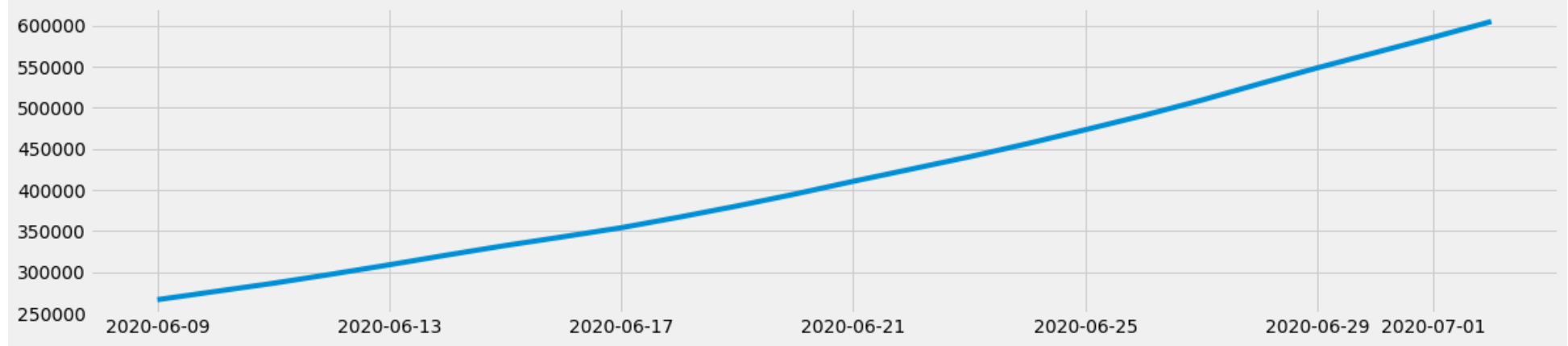

\section{Figure 5}


No. of COVID-19 positive cases predicted for the unlock period

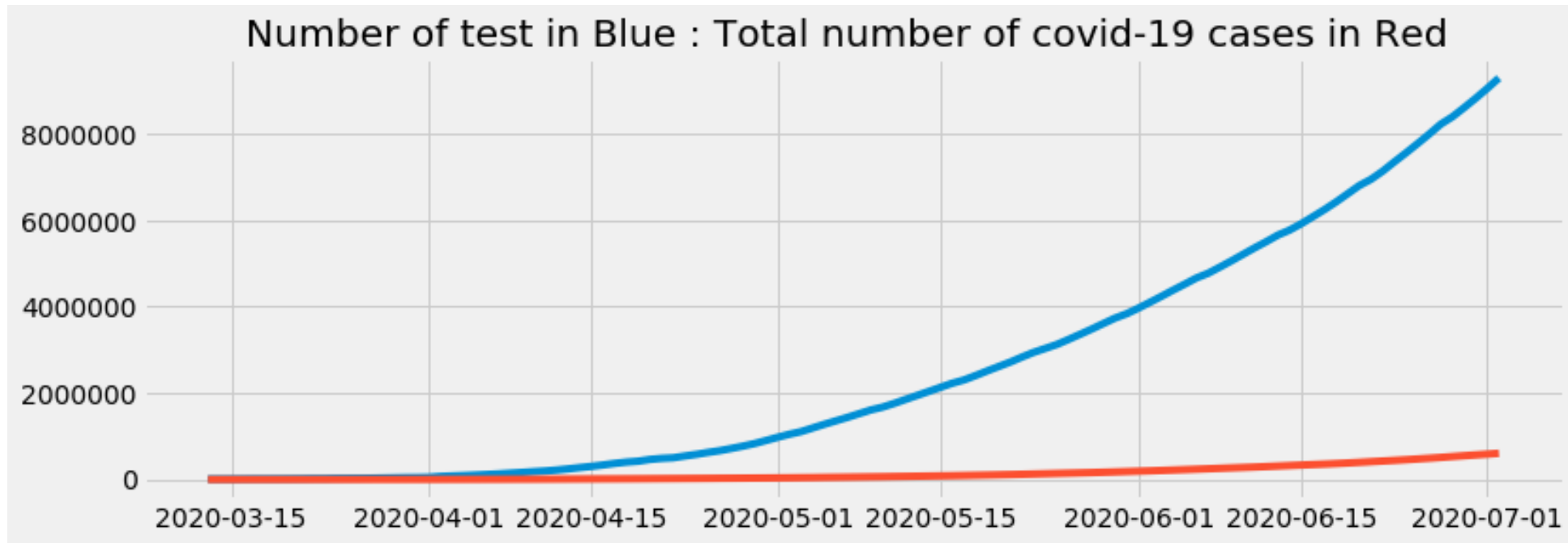

\section{Figure 6}

Number of tests conducted VS Number of positive COVID-19 Cases

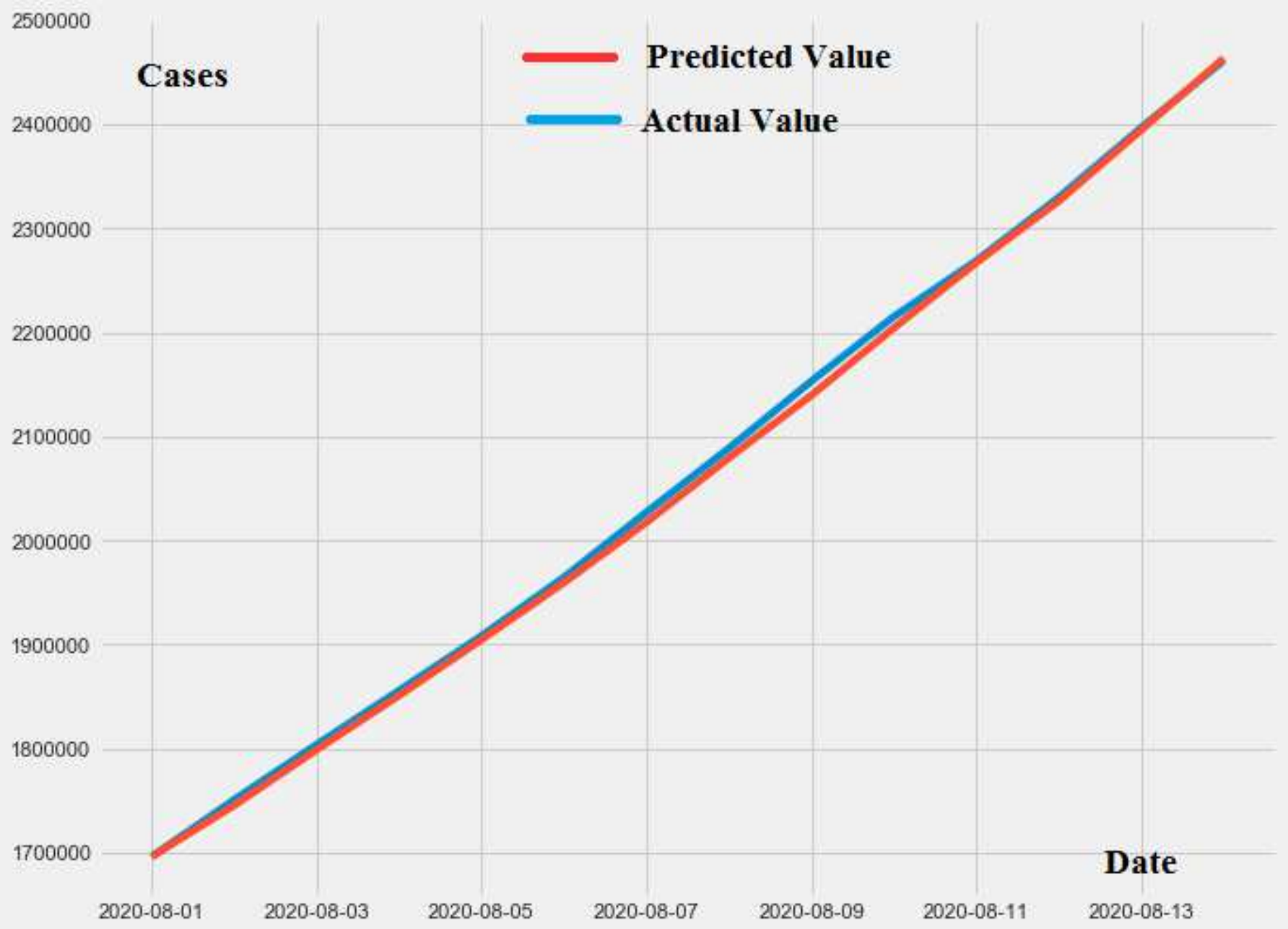

Figure 7 
Number of Positive cases predicted by the proposed model vs Actual cases, from 1st Aug to 14th Aug,2020 